\title{
technology feature
}

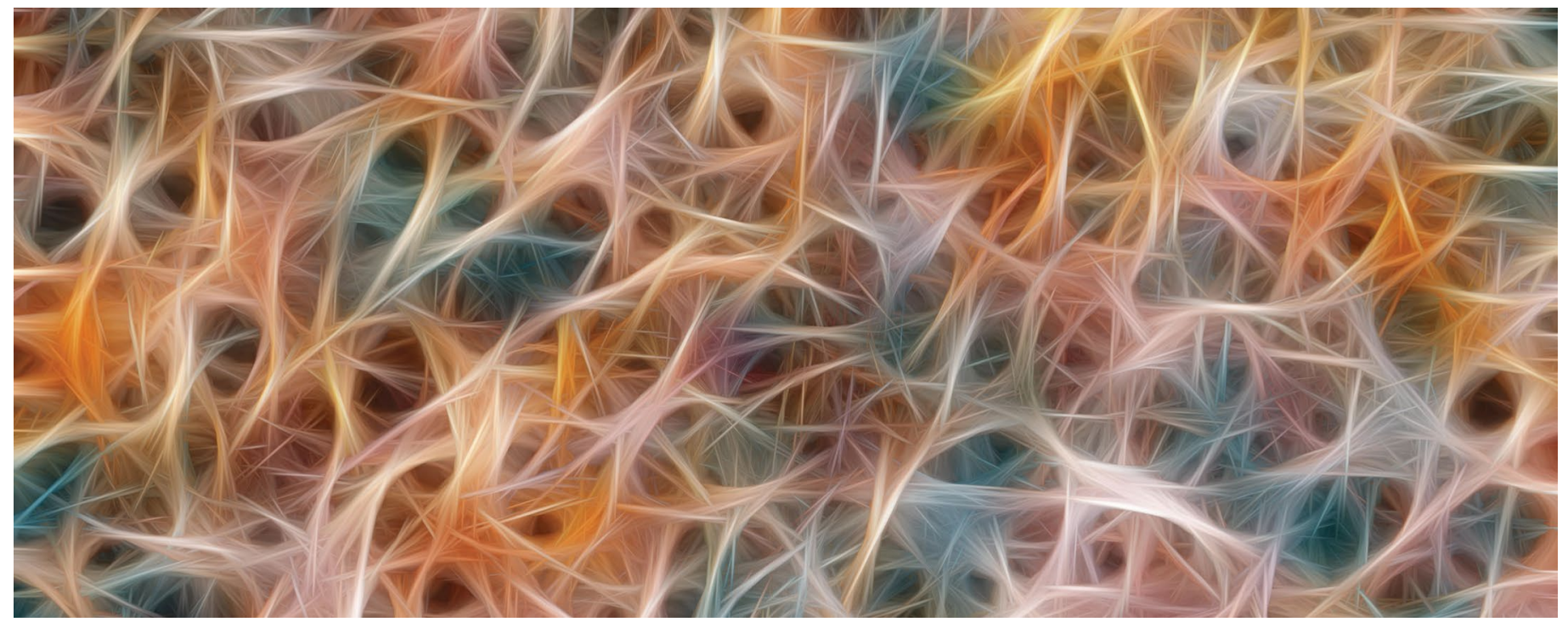

Credit: maksimee / Alamy Stock Photo

\section{The many ways to map many brains}

Whether the animal has a few hundred neurons or a few billion, mapping the 'brain' is a complex endeavor for which there are several different tacks one might take. Data collection continues on.

\section{Ellen P. Neff}

rains come in many shapes, sizes, and degrees of complexity-even the seemingly simplest of organisms need a means to perceive their environment and respond to it. The human brain receives and transmits information across billions of neurons, while model invertebrates such as Caenorhabditis elegans and Ciona intestinalis make do with just a few hundred. When it comes to understanding brains, no matter the species, there's a lot to learn. "I would say we know a lot about a little," says Partha Mitra, "There's all this variation in biology, yet we're seeking common principles-and they do exist."

Mitra is a physicists-turnedneuroanatomist at Cold Spring Harbor Laboratories in New York and the principle investigator of the Brain Architecture Project, a collaborative effort to create cell type and connectivity atlases for mouse, marmoset, zebra finch, and, ultimately, human brains. His effort is one of several to 'map' the minds of different animals. Some projects are large in scale and involve dozens of collaborators and partner institutions, such as the tool building- and database generating- efforts sponsored by the latest US National Institutes of Health (NIH) BRAIN Initiative. Mouse and man tend to be a focus of such work, but other model organism communities have been tackling brain mapping in their own animals - with an eye towards sharing what they find in open databases and web portals for others to take advantage of (Table 1).

Some projects are focused on patterns of connectivity, at the scale of individual neurons or over larger networks across
Table 1 | Data, data, data. A (incomplete) selection of brain atlases, maps, and other databases for different model organisms.

\begin{tabular}{ll}
\hline Caenorhabditis elegans & Worm Wiring \\
& Worm Atlas \\
\hline Drosophila & Virtual Fly Brain \\
& Fruit Fly Brain Observatory \\
\hline Zebrafish & Max-Planck Zebrafish Brain Atlas \\
& Z Brain Atlas \\
& Zebrafish Brain Browser \\
\hline Marmoset & Marmoset Brain Connectivity Atlas \\
\hline Macaque & Marmoset Gene Atlas \\
\hline Mouse & NIH Blueprint NHP Atlas \\
& Mouse Connectome Project \\
& Allen Mouse Data Portal \\
\hline Mouse Brain Architecture \\
\hline EPFL Blue Brain Cell Atlas \\
Mouse Brain Synaptome Map \\
MouseLight
\end{tabular}




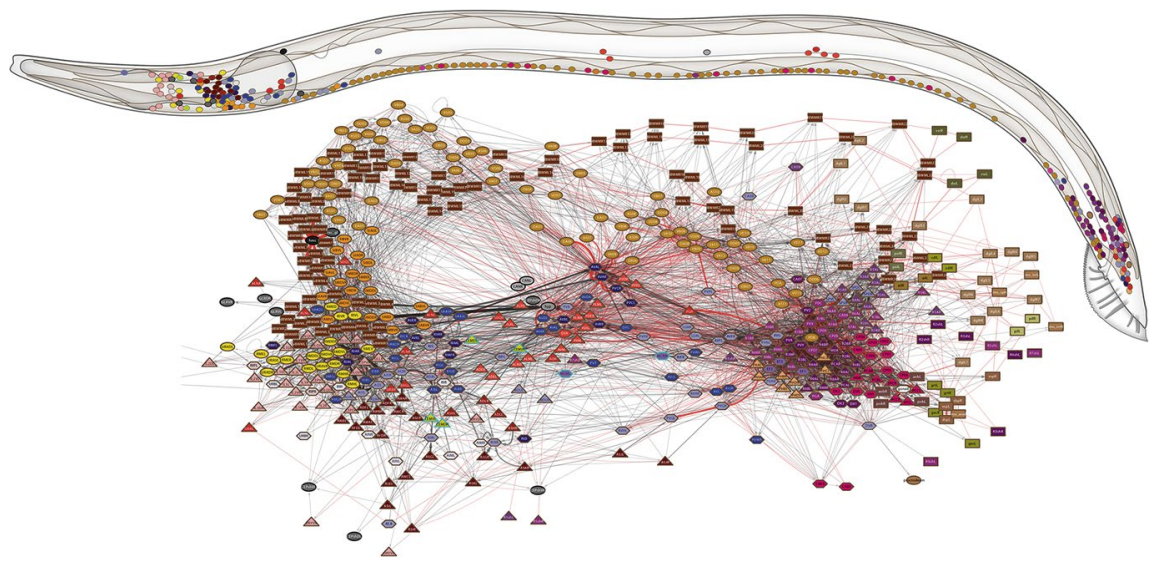

Do the worm | The connectome constructed for the male C. elegans. Image adapted with permission from ref. ${ }^{2}$ different brain regions. Others are looking at the smallest scale-that of synapses, the junctions between neurons that relay information between the cells. Classifying the cell types of the neurons themselves is also an emerging priority. Some technologies to map the brain are new; others, more classic approaches. In the end, there are many ways to think about the brain, and many animals in which to study it. Each has its own advantages and disadvantages, but there's a common theme: a desire to figure out what's there in each animal in the first place.

\section{Making connections}

One approach to mapping the brain is to follow the neurons. The first such wiring diagram of neuronal connections in an organism, known as the connectome, was completed by hand in 1986 for the model worm, C elegans ${ }^{1}$. The team in Sydney the hermaphrodite sex of the worm, fixed those samples, and photographed each with a transmission electron microscope (TEM). The resulting images then had to be physically printed, the locations and other morphological details about the neurons annotated by hand, and the three dimensional volume of the nervous system reconstructed manually in lab notebooks.

For simple animals, the approach is still more or less the same. The biggest difference these days? "Everything's done digitally," says Steven Cook, a C. elegans researcher currently at Columbia University in New York who recently reconstructed connectomes for both worm sexes: the hermaphrodite and the male ${ }^{2}$. Gone are the days of developing film and marking up physical images from photographic micrographs, notes Kerrianne Ryan from Brenner's lab manually cut serial sections of
Dalhousie University in Halifax, who in 2016 completed the connectome of the larval form of the basal chordate Ciona intestinalis ${ }^{3}$. EM images are captured directly into the computer, and various programs can assist with montaging and aligning all the sections. Neurons, however, still have to be traced by hand-or rather, mouse click.

"It was a long process," says Ryan of the Ciona connectome. "Certainly not quite as tedious as the original C. elegans work, but still requiring a lot of manual work and a lot of patience." Constructing Ciona's connectome involved over 5000-nanometerthick sections, cut by hand by technician Zhiyuan Lu. The resulting diagram contained 200 neurons, distinguishable between 177 in the central nervous system and an additional 23 in the peripheral nervous system and with notable asymmetry and local subnetworks. "We thought actually that the connectome would probably be a lot simpler than it ended up being," says Ryan. There might be more nuance to what was thought of as a simple animal with few behaviors, she says.

C. elegans had new details to reveal three decades later as well. The new diagrams revealed previously unaccounted for neurons, says Cook. The hermaphrodite has 302 neurons, while the male has a few more: 385 . As the male transitions from its do indeed transdifferentiate or divide to produce neurons, he notes.

Simplicity has its value. "I find it very satisfying to know where C. elegans begins and where it ends," says Cook. "If you can know everything that's there, you can start to understand what it's doing," says Ryan, without missing the forest for the trees. "When you have fewer trees, you can see more of what's going on," she says. "When you suddenly have so many trees, you can miss things."

\section{In to the forest}

Nevertheless, efforts have also been underway to tackle the connectomes of brains with thousands and millions neurons to trace. The fruit fly, Drosophila melanogaster, is a model invertebrate but with a bit more complexity to wade through in its nervous system than worms or sea squirts. Aided by a custom EM array and high-throughput imaging pipeline, Davi Bock and colleagues at the Howard Hughes Medical Institute Janelia Research Campus recently imaged 100,000 neurons in the $\mathrm{fly}^{4}$. They started tracing the connections, initially with a subset of neurons in the fly's mushroom body, structures in the fly brain that play a role in olfactory learning and memory.

The brain of the larval zebrafish is of comparable size to that of a fruit fly, but the vertebrate fish has an added benefit, says Michael Kunst of the Max Planck Institute of Neurobiology: the animal is transparent. That means the entire brain can be viewed simultaneously-no dissections needed. "We can just look at the fish," he says, "It's a huge time saver." Rather than working with electron microscopes, which require physically sectioned samples, Kunst uses whole larvae and confocal microscopes. In recent years, these have been getting faster, larval to adult form, some non-neural cells

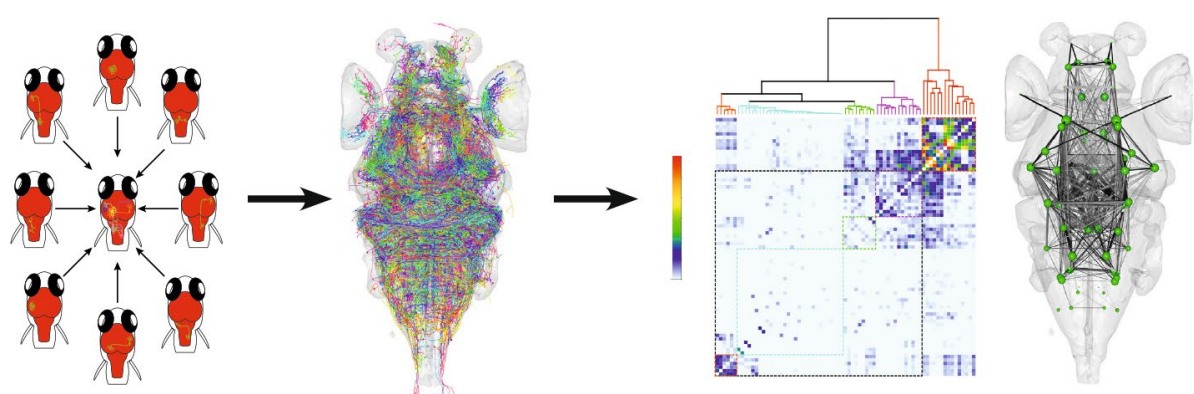

In process | Schematic of the approach to constructing a zebrafish wiring diagram. Credit: M. Kunst 
enabling high-resolution images to be captured in shorter amounts of time.

To visualize individual neurons and follow their paths through the brain, Kunst and others attempting to map more complicated brains take advantage of fluorescent reporters that will light up subsets of neurons. The first iteration of a zebrafish connectome traced 2,000 green fluorescent protein-labeled neurons in the larval brain ${ }^{5}$. Not unlike the classic Golgi method of staining neurons silver, Kunst explains it was a stochastic process-he and his colleagues used the GAL4/UAS binary expression system to randomly create different zebrafish lines with different subsets of glowing neurons that can then be visualized and combined together against a standard larval brain. With 98,000 neurons to go, Kunst is looking to outsource some of the effort, sharing his methodology and fish lines with other labs.

Moving to mouse, the scale of constructing connectomes becomes a bigger challenge. "The mouse brain has 100 million neurons and obviously, to achieve that goal is really long term," says Hongwei Dong, a researcher at the University of Southern California and principle investigator of the Mouse Connectome Project (MCP), a multiinstitute collaboration that aims to define neural network as well as cell types in the mouse brain. The mouse brain, however, can be thought of at different scales, says Michael Bienkowski, a neuroanatomist working on the MCP. At one scale, it may indeed be a collection of millions of neurons, he notes. "But each of those neurons can be grouped into subpopulations-groups of neurons which we call cell types. And then those cell types make up regions, those regions are connected together to form brain-wide networks. And so trying to understand that relationship across scales is critical to understanding how the brain is going to work."

The collaborators at the MCP are taking a systematic approach, moving through different regions of the mouse brain with fluorescent tracer injections that label different neurons. Brains are fixed, sliced and stained, and the fluorescent paths visualized. In 2014, the project published an initial cortical connectome map ${ }^{6}$; they have since been working through other parts of the cortex, the hippocampus, and then on to the amygdala and the brain stem. Mitra's Brain Architecture Project is also taking a more mesoscale view, using fluorescent tracers to label cells across slices of whole brains of several different species and visualize different areas with a classic histological pipeline borrowed from clinical pathology.

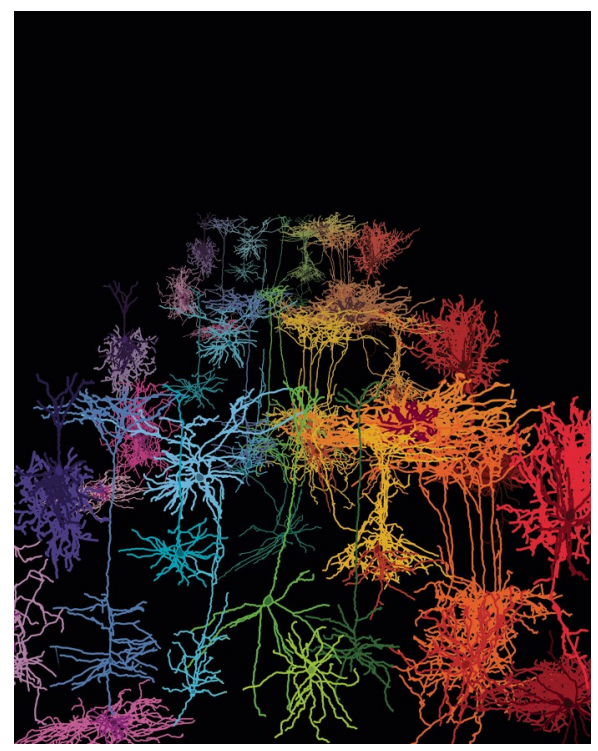

Just the type | Neuronal cell types. Credit: B. Rossi, The Allen Institute

\section{Identify your cells}

Connectivity, however, is but one way to classify neurons in the brain; other characteristics includes its cellular morphology, electrophysiology, and gene expression patterns. "A big focus for the future is trying to integrate more of these different characteristics," Bienkowski says. He and Dong for example recently combined connectome information with mouse brain gene expression data from the Allen Institute, a bioscience research organization in Seattle; analyzing the results of the two parameters together allowed them to further subdivide the hippocampus into finer regions than had previously been recognized ${ }^{7}$.

Identifying, classifying, and categorizing neurons by cell type in mice, marmosets and humans is a major goal of the NIH BRAIN Initiative Cell Census Network. One group involved, the Allen Institute, is currently building a mouse brain cell type atlas, collecting and compiling data about molecular, anatomical, and physiological characterizations of neuronal cell types in mouse. Given the sheer size of the mouse brain, they are taking two tacks, says project lead Hongkui Zeng: go deep, and go broad.

'Depth', says Zeng, means focusing in on just one region but using multiple means; Zeng's efforts are currently directed at the mouse visual cortex. Acquiring morphoelectrical data relies on classical methods: they create CRE driver lines that will label different neurons with fluorescent proteins, thus providing a means to separate out individual neurons for single-cell patch clamp recording, a method used to determine its electrophysiological and morphological detail. In work published in Nature Neuroscience, she and colleagues recorded electrical activity for 2,000 mouse neurons in the visual cortex and established the morphology for 500 of those ${ }^{8}$.

To go broad and catalogue cell types across the entire mouse brain, they are making use of more recent advances in single-cell transcriptome sequencing ${ }^{9}$. The initial iteration of the Mouse Cell Type Atlas might not be complete, notes Zeng, "but it will give us a very, very good first idea about cell types across the whole CNS."

Single-cell RNA sequencing is a rapidly improving and scalable technique, and there are many versions from which to choose; with multiplexing and 'barcodes,' gene expression from single cells can now be measured while maintaining the anatomical integrity of tissues, notes Bienkowski. "It's not just seeing what genes are expressed in the cells, but where those cells exist in the tissue." Each RNA- seq approach brings something slightly different he says, "but I think they are all headed towards the same goal, which is trying to visualize as many cells as possible and as many different genes at the same time."

Single-cell RNAseq techniques are of interest to other model organism communities as well. For the duo of organisms with completed connectomes, transcriptomics is a next step. "That's very important to actually understand what we're looking at. You can't look at it only in terms of wiring, you need to know what the cells are, what they're doing, what they're expressing," says Ryan. Others in the small Ciona community have started on such work, while the CENGEN Project aims to do the same for the worm. Kunst would like to see it for zebrafish as well.

Cell type data does come with complexity. "When you look at the relationships across different cells, they don't fall cleanly into specific types," say Zeng. There are transitions and gradients between cells, as well as intermixing of cell types with different cell states. Hence, a single approach won't create the complete picture.

\section{Assembly required}

Future challenges indeed include collecting data at single-cell resolution says Dong, but, he notes, "We should consider this a good problem." The technology exists to label neurons, and now even to optically clear the brain to reduce interference from tissue. "The really challenging part still is, I think, the imaging speed-the throughput," he says. Data collection continues, at different scales and in different animals and 


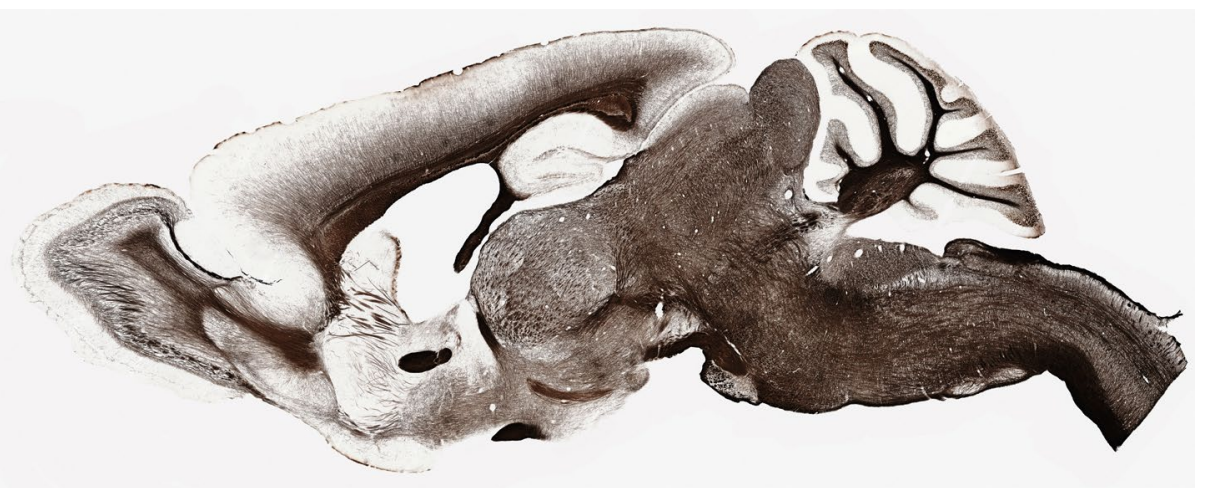

Mice on the brain | A myelin-stained mouse brain section. Credit: P. Mitra

spurred on by improving technologies-for collecting data and storing it (Box 1). "You can collect data much faster than ever before, at much higher resolution," says Dong. "But the challenging part is: how can you make sense of all this data? What does it tell you?"

Connectomes, even when complete, won't by themselves reveal what those neurons are actually doing.

"I like to think about brain maps as hypothesis-generating platforms," says Cook. "The brain maps are such high resolution, but they're also just a static snapshot." The C. elegans community may know where neurons are and how they articulate with one another, but there's still much to learn. "Variability from animal to animal is still, I believe, a completely unresolved issue," he says, as are the mechanisms that drive synaptic specificity and the means by which cells adopt their terminal states. In zebrafish there's been work on visual processing and the spinal cord says Kunst, but "there's just so much in between that people don't really understand."

For C. elegans and Ciona, constructing connectomes from multiple animals at different developmental stages is a future goal. "Looking at multiple individuals can tell you what seems to be more stable," says Ryan, "It might give you insight into what's more required, or what's more fundamental to the behaviors you are going to see." The value of comparison extends beyond individuals of the same species. "As more and more wiring diagrams are emerging, we're able to compare fundamental aspects about how neurons connect to each other," says Cook. "Are the properties of how a worms' brain is wired similar to a fly; a zebrafish; a mouse; a human?" Details concerning how axons grow or how its synapses are formed and maintained are applicable across systems, he says, but with smaller organisms with fewer neurons, there's less ambiguity about what is being observed. Though it's still early days, the increasing number of connectomes becoming available is generating interest in comparative connectomics. "The more we know about different brains from a perspective of evolution, it's nice to see how evolution has accomplished the same thing in different animal models," says Kunst.

\section{Box 1 | Can compute}

Technology developments, spurred on by large, coordinated efforts like the NIH BRAIN Initiative, are allowing researchers to collect brain data faster and in new ways. But better microscopes and new sequencing methods are only part of the picture; whatever the data is, it has to end up somewhere. Just consider the computational tools, says Mitra, "the fact that we can simply store these datasets." Sequencing data exist in gigabyte scales, but imaging data can push into terabyte ranges and well beyond. The mouse brain connectivity data set in the Brain Architecture project, for example, reached 2 petabytes. But storage prices have dropped while computational power has increased, Mitra says.

Computers are also aiding construction and initial analysis. "A lot of these data sets are just too large for any one person to look at within a reasonable amount of time," says Bienkowski. Here, machine learning can help scientists sift through the data sets and find patterns that might merit a closer look. For their work on the synaptome, Grant's lab for example has developed a machine learning algorithm to classify one billion mouse synapses into 37 different types. ${ }^{10}$ Though there are hundreds of proteins expressed on synapses, he targeted just two
Kunst would also like to see work on older fish-that could bring social behavior into play, but will bring added technical challenges as the animals become opaque as they age. Plus, "bigger fish, longer imaging time," he says. Most mouse work meanwhile is on a young adult male 'black 6' mouse; an important baseline but one that doesn't reveal detail about disease or development, says Dong.

Connecting structures to function is an important follow-up step after data is recorded. From a completed connectome, you can start to see whether behaviors are as simple or complex as the wiring might suggest, says Ryan. The connectome might show a simple pathway from A to B to C, but does behavior follow? For example, the locations of Ciona's photoreceptors are known; with the connectome, researchers can look downstream at motor neurons and make predictions about how the animal should behave in response to a stimulus and then test whether it does so. Kunst has been collaborating with researchers at Janelia to correlate whole-brain function in response to different visual stimuli with neurons in his zebrafish atlas to begin the construction

with fluorescent proteins, and then set the algorithm to work classifying those along with physical details such as shape and size. The transgenic mice needed are easy to make and high speed microscopes could complete scans in less than 24 hours, he says, but nevertheless computer runs could take weeks. "The major bottlenecks were all computational.” Adding fluorescent labels to additional proteins increases the possible combinations to be classified even more, though there are likely constraints to the numbers of complexes that can be formed, Grants says; the architecture needs to be understood better, but it's not random.

Mitra has been applying machine learning to aid as a 'virtual anatomist,' automating the workflow of stitching 2D images into 3D volumes for his project's different brains. "If you have small amounts of data, you can have someone run an algorithm and verify it by pulling up the images and do a lot of human labor intensive work," he says "But when you have these datasets which are a million times larger, that human in the loop component really has to be drastically reduced. It is more and more pressure to be more automated, in a more in the modern AI sense. It's still evolving, but that's where the challenges are and we've made significant progress." 
of a functional diagram that can reveal details about how information flows in thebrain.

Even as a wiring diagram of every single neuron remains a future goal for many of the different model organisms, establishing such larger networks of connectivity between brain regions can still be informative and aid predictions about the role of different parts of the brain. If an area known to be involved in, say, spatial navigation is connected to another region with unknown function, that can indicate what that unknown region might be doing, says Bienkowski. "When you understand the connectivity of an area, you can form some hypotheses about function." The Mouse Connectome Project is still collecting data, but the group is interested in starting to build computational models of their wiring diagrams so far. "I think that could tell us a lot about what the biological networks actually are doing if we have a way to simulate connectivity."

Another view towards function might come from synaptic detail.. The molecular composition of synapses is determined by the genome; thus, says Seth Grant of the University of Edinburgh, the synaptome could be a means to link genes to the behaviors they encode. His work has shown that different regions of the brain have different distributions of synapse types, suggesting different functions. Combined with mouse cortical connectome data, the synapse composition reflected the brain's structural detail. And as synaptomes can be generated for multiple mice, comparisons can be made between wild-type animals and those modeling a particular disease, he says, without having to rely on assumptions about behavioral assays. In future work, he'd like to consider how the synaptome changes at different developmental stages and across lifespans as well.

The added detail provided by cell type catalogues and atlases can bring additional advantages. Once neurons are categorized, researchers can begin to identify genetic markers, says Zeng. From there, genetic tools, such as ever improving AAVs that can introduce proteins to different cells in different animals, can be used to target those specific neurons for, for example, optogenetic experiments to manipulate different neurons in the brain and observe how that affects behavior.

\section{Collection-and collaboration- continues}

The building complete 'parts' lists of animal brains continue, and while timelines for 'completion' vary the different databases out there will continue to grow. That brings its own challenges, says Zeng: how to handle the data (and its metadata) and make it presentable. "In a public database, making everything available in an organized manner, and making sure that the data generated are relevant to each other, is a challenge," she says.

But open databases and an element of cooperation pervade different mapping efforts. "All these new technologies are bringing us together," says Bienkowski, "and getting us to working together, to look at how we're seeing the same thing from different perspectives." The different projects indeed bring together people from myriad different backgrounds, from neuroanatomy to computer science and programing. Much work is ultimately geared towards understanding the human brain and treating its anomalies, but there are also applications for improving the very computers and algorithms that are helping scientists create their different maps-and then some.

"I'm quite happy," says Mitra of the shift towards understanding neuroanatomy, "It's become a viable research field that we are one player in."

Ellen P. Neff

Lab Animal.

e-mail:ellen.neff@us.nature.com

Published online: 23 October 2019

https://doi.org/10.1038/s41684-019-0423-6

References

1. White, J. G., Southgate, E., Thomson, J. N. \& Brenner, S. Philos Trans. R Soc. Lond. B Biol. Sci. 314, 1-340 (1986).

2. Cook, S. J. et al. Nature 571, 63-71 (2019).

3. Ryan, K. Lu, Zhiyuan \& Meinertzhagen, I. A. eLife 5, el6962 (2016).

4. Zeng, Z. et al. Cell 174, 730-743 (2018).

5. Kunst, M. et al. Neuron 103, 21-38.e5 (2019).

6. Hintiryan, H. et al. Nat. Neurosci. 19, 1100-1114 (2014).

7. Bienkowski, M. S. et al. Nat. Neurosci. 21, 1628-1643 (2018).

8. Gouwens, N. W. et al. Nat Neurosci 22, 1182-1195 (2019).

9. Hodge, R. D. et al. Nature 573, 61-68 (2019).

10. Zhu, F. et al. Neuron 99, P781-799.E10 (2018). 